\title{
A questão do nitrato em alface hidropônica e a saúde humana
}

\author{
The nitrate issue in hydroponic lettuce and the human health
}

\author{
Gean Lopes da Luz ${ }^{\text {* }}$ Sandro Luís Petter Medeiros ${ }^{I}$ Paulo Augusto Manfron ${ }^{\mathrm{I}}$ \\ Alan Dischkaln do Amaral ${ }^{\mathrm{I}}$ Liziany Müller $^{\mathrm{I}}$ Mike Guzmán Torres ${ }^{\mathrm{I}}$ Lenise Mentges $^{\mathrm{I}}$
}

\section{- REVISÃO BIBLIOGRÁFICA -}

\section{RESUMO}

A alface (Lactuca sativa L.) destaca-se como a espécie mais produzida em sistema hidropônico. Nesse tipo de cultivo, a maior parte do nitrogênio é fornecida na forma de nitrato, o que pode acarretar acúmulo deste íon nos vacúolos celulares. $O$ teor de nitrato nas plantas depende de fatores genéticos, da disponibilidade desse nutriente no sistema radicular e de fatores ambientais. Diversos estudos indicam que o consumo de nitrato pode ser nocivo à saúde humana, causando metahemoglobinemia e câncer gástrico. Porém, pesquisas recentes discorrem que não há ligação entre nitrato $e$ as doenças citadas, destacando ainda o papel benéfico do nitrato à saúde humana, como protetor contra gastrenterites. Existem indícios de que a presença de certas vitaminas em vegetais inibe uma possível ação negativa do nitrato. Altas concentrações de nitrato em alface cultivada sob hidroponia em países do norte europeu causaram apreensão da comunidade científica e de consumidores brasileiros. Porém, as pesquisas realizadas no Brasil demonstram que, por haver maior disponibilidade de radiação solar, devido às menores latitudes, os teores de nitrato encontrados em alface produzida em hidroponia são bastante inferiores aos limites máximos preconizados pela união européia, indicando não haver risco à saúde humana.

Palavras-chave: Lactuca sativa L., nitrato, metahemoglobinemia, câncer gástrico.

\section{ABSTRACT}

Lettuce (Lactuca sativa L.) is the main vegetable crop produced hydroponically. In this kind of cropping, the majority of nitrogen is provided as nitrate, which may lead to the accumulation of this ion in cells. Nitrate amount in plants depends upon genetic factors, the availability of this nutrient in roots and environmental factors. Several studies have indicated that the consumption of nitrate may be harmful to human health, causing methaemoglobinaemia and gastric cancer. However, recent studies have proposed that there is no link between nitrate and these diseases, and that nitrate may be even good for human health as a protector against gastroenteritis. Some other studies have indicated that the presence of certain vitamins in plants is likely to inhibit possible negative effects of nitrate. High nitrate concentrations in lettuce grown hydroponically in northern European countries have been a matter of concern to the scientific community and Brazilian consumers. Nevertheless, studies have demonstrated that in Brazil, because of higher solar radiation at low latitudes, nitrate amounts found in lettuce produced hydroponically are much lower than the amounts given by the European community, indicating no risk to human health.

Key words: Lactuca sativa L., nitrate, methaemoglobinaemia, gastric cancer.

\section{INTRODUÇÃO}

A alface (Lactuca sativa L.) é a hortaliça folhosa mais consumida no Brasil. Ela é fonte de vitaminas e sais minerais na alimentação da população brasileira e se destaca pelo baixo valor calórico, sendo bastante utilizada em dietas balanceadas e recomendada por nutricionistas. É uma hortaliça folhosa com grande importância alimentar pelo fato de ser tradicionalmente consumida crua, suprindo o organismo com a totalidade de seus componentes nutricionais, tais como: vitaminas

IDepartamento de Fitotecnia, Universidade Federal de Santa Maria (UFSM), 97105-900, Santa Maria, RS, Brasil. E-mail: geanluz@hotmail.com.*Autor para correspondência. 
A, $B_{1}, B_{2}$, C e sais minerais de ferro e cálcio (OHSE, 1999). A alface é a espécie mais cultivada e adaptada ao sistema hidropônico, destacando-se por seu ciclo curto, possibilitando retorno mais rápido do capital investido.

Nas hortaliças, especialmente folhosas, 0 nitrogênio desempenha papel fundamental no crescimento e no rendimento dos produtos colhidos. Um adequado suprimento de nitrogênio está associado à alta atividade fotossintética e ao crescimento vegetativo vigoroso (CASTELLANE, 1994; FILGUEIRA, 2000). Em alface, doses elevadas de nitrogênio proporcionam maior massa e maiores acúmulos de macronutrientes nas folhas (PEREIRA et al., 1989; ALVARENGA et al., 2000; FERREIRA et al., 2000). Em sistema hidropônico, o nitrogênio é fornecido basicamente na forma de nitrato, uma vez que o amônio em altas concentrações na solução nutritiva é fitotóxico para a planta de alface, reduzindo o rendimento e a qualidade visual da cultura (BOON et al., 1990; FAQUIN et al., 1994; FAQUIN \& FURTINI NETO, 1996; FURLANI, 1998). A capacidade de acúmulo de nitrato no vacúolo celular é de caráter genético, porém, grandemente influenciada por outros fatores, tais como: disponibilidade do íon na solução nutritiva, intensidade luminosa, disponibilidade de molibdênio, temperatura, umidade relativa do ar, sistema de cultivo, época de cultivo e hora de colheita, sendo os dois primeiros os mais importantes (MAYNARD et al., 1976; FAQUIN \& FURTINI NETO, 1996; ANDRIOLO, 1999).

Atualmente, existe grande preocupação por parte dos consumidores, e até mesmo dos produtores, com a qualidade dos alimentos, destacando as questões sanitária, organoléptica e nutricional como as principais. Entre as características nutricionais estudadas, a concentração de nitrato em alface cultivada em hidroponia destaca-se pela possibilidade de ser nociva à saúde humana. Esse aspecto, discutido em diversos trabalhos científicos, apresenta divergências e tópicos importantes que se complementam, mas que têm sido discutidos separadamente. Dessa forma, o objetivo deste estudo é a compilação e a divulgação dos conhecimentos sobre o assunto, tanto para os pesquisadores quanto para os produtores e consumidores dessa cultura, analisando se o consumo de alface hidropônica representa risco à saúde da população brasileira.

\footnotetext{
Nitrogênio - nitrato, nitrito e amônio

O nitrogênio é um elemento essencial. Ele exerce, nas plantas, função estrutural na formação de aminoácidos, proteínas, enzimas, coenzimas, vitaminas e pigmentos, como nas moléculas de clorofila, determinando o crescimento e o desenvolvimento das
}

plantas e a produtividade dos cultivos. Seus efeitos são ligados ao aumento da área foliar, sendo utilizado em grandes quantidades em adubações de folhosas, como a alface, em que a parte comercial é a parte vegetativa (MAYNARD et al., 1976; TAIZ \& ZIEGER, 2004).

No solo, a maior parte do nitrogênio absorvido pelas plantas está na forma de nitrato. No sistema hidropônico, o nitrogênio também é fornecido em sua maior parte sob a forma de nitrato, uma vez que o amônio fornecido acima de $15 \%$ do nitrogênio total da solução, segundo FAQUIN et al. (1994), e 30\% do nitrogênio total da solução, segundo ZITO et al. (1994), pode ser fitotóxico, causando reduções no rendimento e na qualidade das plantas.

Antes de constituir compostos orgânicos formando aminoácidos, proteínas e outros compostos nitrogenados, o nitrato absorvido pelas raízes deve ser primeiramente reduzido para amônio. Esta redução, em sua maior parte, ocorre nas folhas e em duas etapas: a primeira no citoplasma, em que o nitrato passa para nitrito, processo mediado pela enzima nitrato redutase; a segunda nos cloroplastos, em que o nitrito passa para amônio, por ação da enzima nitrito redutase. $\mathrm{Na}$ primeira etapa, o agente redutor é o $\mathrm{NADH}^{+}$, originado na respiração, e na segunda etapa, nos cloroplastos, o agente redutor é a ferredoxina, cujos elétrons são originados no fotossistema I da fase fotoquímica da fotossíntese (FAQUIN \& ANDRADE, 2004). Na seqüência, o amônio combina-se a compostos (ou cadeias) orgânicos, formando glutamina e, a partir dela, outros aminoácidos. Os aminoácidos são as unidades básicas na formação de proteínas, as quais participam dos processos metabólicos das plantas, tendo papel funcional e estrutural (TAIZ \& ZIEGER, 2004).

Por que a planta acumula nitrato?

O acúmulo de nitrato nos tecidos vegetais ocorre quando há desequilíbrio entre a absorção e a assimilação desse íon, sendo que as quantidades excedentes são estocadas nos vacúolos para serem assimiladas posteriormente (ANDRIOLO, 1999).

Os principais fatores que afetam o acúmulo de nitrato nas plantas são de origem genética, ambiental, da forma de fornecimento do nitrogênio (quantidade e proporção) e da quantidade de molibdênio fornecido. Entre as variáveis genéticas, citase que as cultivares de alface de folhas lisas tendem a acumular mais nitrato que as de folhas crespas (OHSE, 1999). Uma grande variabilidade de conteúdo de nitrato tem sido observada em diferentes espécies vegetais, assim como dentro de uma mesma espécie (CARDENAS - NAVARRO et al., 1999). A acumulação é provocada geralmente por algum estresse ambiental em que a taxa 
fotossintética da planta é afetada, especialmente em situações de baixa incidência de radiação solar associada à alta disponibilidade desse nutriente em nível radicular. Tal fato provoca diminuição da atividade da enzima nitrito redutase pela falta de energia para formação de $\mathrm{NADH}^{+}$. Um ligeiro acúmulo de nitrito ocasiona a inibição da atividade da enzima nitrato redutase, resultando em acúmulo de nitrato nos vacúolos celulares (HUFTON et al., 1996).

Como fatores ambientais, pode-se destacar a intensidade de radiação luminosa e a temperatura do ar, entre outros fatores que afetam a fotossíntese. A irradiância elevada reduz o acúmulo de nitrato. Isso foi mostrado por meio de plantas cultivadas sob circunstâncias naturais de luz, durante as estações do ano, ou nas experiências sob radiação controlada, conforme CARDENAS-NAVARRO et al. (1999). A redutase do nitrato é uma enzima induzível. A luz pode, então, influenciar o metabolismo do nitrato também por meio de efeitos sobre a síntese dessa enzima (OHSE, 1999).

Quanto à forma de fornecimento de nitrogênio, ressalta-se que a quantidade de nitrato fornecido tem função linear com a quantidade de nitrato acumulado na planta. Em contrapartida, a utilização de parte do $\mathrm{N}$ total como amônio, além de evitar um gasto energético de 15 a 16 ATP por mol de $\mathrm{NO}_{3}$ - requerido pela enzima nitrato redutase, reduz o acúmulo de nitrato. Todavia, plantas nutridas com amônio têm, usualmente, a produção de massa de matéria seca reduzida em 15 a $70 \%$ em relação a plantas supridas com nitrato (SALSAC et al., 1987).

A deficiência de molibdênio promove acúmulo de nitrato na planta, uma vez que esse nutriente é um importante componente da enzima nitrato redutase, auxiliando, desse modo, na assimilação do nitrato pela planta (ANDRIOLO, 1999).

Conteúdo de nitrato em alface cultivada em hidroponia no Brasil

Existem muitas divergências na bibliografia nacional quanto aos teores de nitrato em alface hidropônica. Estudando o efeito de sistemas de cultivo na produtividade e no acúmulo de nitrato em cultivares de alface em Jaboticabal, SP, MONDIN (1996) observou teor de 581,6mg de $\mathrm{NO}_{3}{ }_{3}^{-} \mathrm{kg}^{-1}$ de matéria fresca (MF), em alface cultivada em hidroponia, e de $1.129,9 \mathrm{mg}$ de $\mathrm{NO}_{3}^{-}$ $\mathrm{kg}^{-1}$ de MF nos cultivos de campo, destacando-se o maior teor de nitrato no cultivo em campo que no cultivo hidropônico.

Avaliando a qualidade nutricional e o teor de nitrato da alface sob cultivo hidropônico em experimento realizado na primavera, em Santa Maria,
RS, OHSE (2000) encontrou teores de 258,8mg de $\mathrm{NO}_{3}^{-}$ $\mathrm{kg}^{-1} \mathrm{MF}$ em alface sob cultivo hidropônico e 236,6mg de $\mathrm{NO}_{3}^{-} \mathrm{kg}^{-1}$ de $\mathrm{MF}$ em alface cultivada à campo sob cultivo convencional, observando que os teores de nitrato ficaram bastante próximos entre os dois cultivos. Já em estudos realizados por BENINNI et al. (2002), coletando amostras de alface no mercado de Londrina, $\mathrm{PR}$, e analisando o teor de nitrato em alface cultivada em sistemas hidropônico e convencional no período de inverno, encontraram, para o sistema hidropônico, teores médios de $1.588 \mathrm{mg}$ de $\mathrm{NO}_{3}{ }^{-} \mathrm{kg}^{-1}$ de $\mathrm{MF}$, com o máximo de $2.568 \mathrm{mg}$ de $\mathrm{NO}_{3}{ }^{-} \mathrm{kg}^{-1}$ de $\mathrm{MF}$, e o mínimo de 471mg de $\mathrm{NO}_{3}^{-} \mathrm{kg}^{-1}$ de $\mathrm{MF}$, e para cultivo convencional de campo

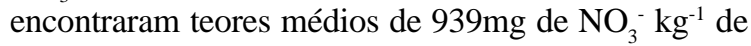
$\mathrm{MF}$, com máximo de $1.910 \mathrm{mg}$ de $\mathrm{NO}_{3}^{-} \mathrm{kg}^{-1}$ de $\mathrm{MF}$ e mínimo de $26 \mathrm{mg}$ de $\mathrm{NO}_{3}^{-} \mathrm{kg}^{-1}$ de MF. Ressalta-se, portanto, o maior teor de nitrato no cultivo hidropônico que no cultivo em campo. Analisando a produtividade, o acúmulo de nitrato e o estado nutricional de cultivares de alface em hidroponia, em função de fontes de nutrientes no outono no município de Viçosa, MG, FERNANDES et al. (2002) citam teores médios que variaram de 500 a $960 \mathrm{mg}$ de $\mathrm{NO}_{3}^{-} \mathrm{kg}^{-1}$ de $\mathrm{MF}$, destacando-se os baixos teores encontrados.

Avaliando o efeito da condutividade elétrica da solução nutritiva no acúmulo de nitrato em alface cultivada em sistema hidropônico no inverno, em Londrina, PR, FILGUEIRAS et al. (2002) encontraram teores variando de 442 a $763 \mathrm{mg}$ de $\mathrm{NO}_{3}{ }^{-} \mathrm{kg}^{-1}$ de MF. Os teores de nitrato responderam linearmente ao aumento da concentração da solução nutritiva, porém, permaneceram baixos mesmo no inverno e sob alta condutividade elétrica.

No entanto, na Europa, principalmente ao Norte do continente, onde há menor quantidade de radiação solar disponível para as plantas, os teores de nitrato em alface hidropônica são mais elevados, alcançando no inverno teores de até $6.000 \mathrm{mg}$ de $\mathrm{NO}_{3}^{-}$ $\mathrm{kg}^{-1}$ de MF (BOON et al., 1990).

As divergências apresentadas nas bibliografias, possivelmente, se devem ao fato de os teores de nitrato serem muito sensíveis à metodologia adotada e, portanto, podem variar por diversos fatores, tais como o procedimento de determinação, a parte da planta amostrada, o horário da amostragem, entre outros.

Ao analisar os teores de $\mathrm{NO}_{3}{ }^{-}$em alface de cultivo hidropônico e comparar os procedimentos da destilação, do ácido salicílico e da mistura redutora contendo zinco com o procedimento da coluna redutora contendo cádmio (método oficial de determinação de nitrato em carnes e demais alimentos no Brasil), MANTOVANI et al. (2005) citam que os procedimentos 
do ácido salicílico e da mistura redutora contendo zinco superestimam os teores das amostras, e o método da destilação se aproxima bastante dos teores da coluna redutora contendo cádmio. Esses resultados demonstram que o procedimento utilizado na determinação dos teores de nitrato pode ser determinante dos níveis dos resultados encontrados, ressaltando a importância da aplicação da metodologia oficial para padronização dos resultados.

Em estudos fisiológicos, principalmente de metabolismo do nitrogênio, além da observação da metodologia química adotada, a segmentação das partes pode ser fundamental na interpretação dos resultados. Na análise de nitrato, $\mathrm{N}$-amino e açúcares livres na alface, é recomendada a separação de folhas e caule da parte aérea, por apresentarem grandes diferenças (COMETTI et al., 2004). KROHN et al. (2003) encontraram maiores teores de nitrato nas folhas novas em relação às folhas maduras e COMETTI et al. (2004) encontraram teores crescentes de nitrato para limbos foliares, nervuras foliares e caule.

O horário de coleta das amostras para análise também merece atenção, pois plantas de alface colhidas ao amanhecer apresentam maiores teores de nitrato que as colhidas ao entardecer (FAQUIN et al., 1994; KROHN et al., 2003). Tal fato possivelmente ocorre devido à falta de luminosidade durante a noite $\mathrm{e}$ o amanhecer, o que inibe a atividade fotossintética e a redução do nitrato a amônio, resultando em acúmulo nos vacúolos celulares. Quando há disponibilidade de radiação solar, durante o dia, as enzimas nitrito e nitrato redutase são ativadas, reduzindo o nitrato acumulado a amônio e diminuindo os teores de nitrato na planta.

O nitrato e a saúde humana

O nitrato no organismo humano pode se tornar tóxico (PIGNATELLI et al., 1993; JOOSSENS et al., 1996 ; CHUN-YUH et al., 2000). O nitrato ingerido a partir dos alimentos pode ser reduzido a nitrito $\left(\mathrm{NO}_{2}{ }^{-}\right)$ no trato digestivo e ao chegar à corrente sangüínea oxida o ferro $\left(\mathrm{Fe}^{2+} \rightarrow \mathrm{Fe}^{3+}\right)$ da hemoglobina, produzindo a metahemoglobina. A metahemoglobina é incapaz de transportar oxigênio para a respiração celular, o que leva à doença conhecida como metahemoglobinemia, ou doença do "sangue azul" (WRIGHT \& DAVISON, 1964).

Existe também a possibilidade do nitrito combinar-se com aminas formando "nitrosaminas", substâncias caracterizadas como carcinogênicas e mutagênicas. Como o íon nitrato é transformado a nitrito já na saliva, por meio de diversos complexos de redução, presentes na boca, esse nitrito poderia formar nitrosaminas a partir de aminas secundárias, causando câncer gastrointestinal, como foi detectado em animais experimentais recebendo dieta rica em compostos Nnitrosos (MAYNARD et al., 1976).

Os teores de nitrato em alface considerados aceitáveis para o consumo humano variam com a época do ano e não são estipulados nas leis brasileiras, sendo, portanto, adotados índices europeus no Brasil. A Comunidade Européia estabeleceu como limite máximo permitido para alface produzida em casa-de-vegetação teores de nitrato na MF de $3.500 \mathrm{mg} \mathrm{kg}^{-1}$ para o período de verão, $4.500 \mathrm{mg} \mathrm{kg}^{-1}$ para o período de inverno e $2.500 \mathrm{mg} \mathrm{kg}^{-1}$ o limite máximo permitido para alface produzida em campo aberto (McCALL \& WILLUMSEN, 1998). A Organização Mundial para Agricultura e Alimentação (FAO) e a Organização Mundial da Saúde (OMS) estabeleceram como admissíveis as doses diárias de 3,65mg do íon $\mathrm{NO}_{3}^{-}$ e $0,133 \mathrm{mg}$ do íon $\mathrm{NO}_{2}^{-} \mathrm{kg}^{-1}$ de massa corpórea (WORLD HEALTH ORGANIZATION, 1973). O comitê Conjunto FAO/WHO de Peritos em Aditivos Alimentares (JECFA) estabeleceu para o nitrato uma Ingestão Diária Aceitável (IDA) de 0 a 5,0 $\mathrm{mg} \mathrm{kg}^{-1}$ de massa corpórea e uma IDA temporária de 0 a $0,2 \mathrm{mg} \mathrm{kg}^{-1}$ de massa corpórea para o nitrito, recomendando não adicionar nitrito nos alimentos destinados a crianças com menos de seis meses de idade (WORLD HEALTH ORGANIZATION, 1976).

Atualmente, as pesquisas estão se voltando para a desmistificação dos males causados pelo nitrato, surgindo inclusive citações sobre importantes funções do nitrato no organismo humano, principalmente com funções de defesa contra patógenos. Pesquisando casos de metahemoglobinemia no Reino Unido, LEIFERT et al. (1999) mencionam que a última ocorrência registrada em bebês foi em 1972. Além disso, os autores citam que não existem evidências epidemiológicas comprovando a ligação do nitrato dietético com o câncer causado por nitrosaminas. Recentes pesquisas vêm trazendo evidências de que o nitrato dietético apresenta destacado papel benéfico, protegendo a área gastrintestinal contra patógenos que se desenvolvem nos alimentos. Estudos nutricionais e epidemiológicos mostram que a adição de nitrito ao ácido estomacal controla melhor patógenos como Salmonella, Escherichia coli e Helicobacter pylori, que poderiam sobreviver apenas com o ácido estomacal. Além disso, indicam que dietas ricas de saladas e vegetais e, portanto, alto conteúdo de nitrato são protetoras contra alguns tipos de câncer, particularmente câncer gástrico (ADDISCOTT \& BENJAMIN, 2000; ARCHER, 2002). Porém, MCKNIGHT et al. (1999) mostram que experimentos tentando ligar o aparecimento de câncer à dieta rica em nitratos têm demonstrado resultados contraditórios (Tabela 1). 
Tabela 1 - Estudos publicados apresentando evidências contra ou a favor à relação etiológica do nitrato ao câncer gástrico.

\begin{tabular}{|c|c|c|c|}
\hline \multicolumn{2}{|c|}{$\begin{array}{c}\text { Estudos sugerindo a relação do nitrato com a etiologia do câncer } \\
\text { gástrico }\end{array}$} & \multicolumn{2}{|c|}{$\begin{array}{l}\text { Estudos apresentando evidências contra a relação do nitrato } \\
\text { com câncer gástrico }\end{array}$} \\
\hline País & Referência & País & Referência \\
\hline Ucrânia & HILL et al. (1973) & EUA & GELEPERIN et al. (1976) \\
\hline Colômbia & CORREA et al. (1970) & Ucrânia & DAVIES (1980) \\
\hline Colômbia & CUELLO et al. (1976) & Ucrânia & BERESFORD (1985) \\
\hline Chile & ZALDIVAR (1977) & Chile & ARMIJO et al. (1981) \\
\hline Hungria & JUHASZ et al. (1980) & França & VINCENT et al. (1983) \\
\hline Italia & AMADORI et al. (1980) & Ucrânia & FORMAN et al. (1985) \\
\hline \multirow[t]{3}{*}{ Dinamarca } & JENSEN (1982) & Italia & KNIGHT et al. (1990) \\
\hline & & Espanha & GONZALEZ et al. (1994) \\
\hline & & França & POBEL et al. (1995) \\
\hline
\end{tabular}

Fonte: Adaptado de McKNIGHT et al. (1999).

Diversos trabalhos indicam que a formação de compostos N-nitrosos é inibida por vitamina C e E e que o alto conteúdo dessas vitaminas em vegetais pode inibir os possíveis efeitos prejudiciais do nitrato (SOBALA et al., 1989; MIRVISH, 1996; BARRET et al., 1998; WARD et al., 2003). Uma evidência bastante contundente de que o nitrato contido em vegetais não é causador de câncer gástrico é confirmada na observação da dieta de vegetarianos, que consomem três vezes mais nitrato que onívoros e apresentam menores taxas de mortalidade por câncer gástrico (KEY et al., 1996). Além disso, enquanto a incidência mundial de câncer gástrico está em constante declínio, o conteúdo de nitrato na água ingerida e o consumo de vegetais verdes estão aumentando (CORREA \& CHEN, 1994; ADDISCOTT \& BENJAMIN, 2000).

Outro fator a ser considerado é que, em revisão realizada por AVERY (1999), o autor cita que bactérias causadoras de doenças estomacais podem ser responsáveis por vários casos de metahemoglobinemia infantil, previamente atribuídas à ingestão de nitrato por meio da água.

\section{CONCLUSÕES}

Os teores de nitrato em hortaliças são elevados em países com baixa disponibilidade de radiação, como os do Norte da Europa. Porém, nas condições ambientais do Brasil, onde há disponibilidade de radiação suficiente para uma boa assimilação do nitrato, esse não se acumula em níveis elevados nas plantas, como comprovam os dados observados na bibliografia, que ficam bem abaixo dos preconizados pelas leis européias. Desse modo, a alface cultivada em hidroponia sob as condições climáticas do Brasil, com as soluções nutritivas indicadas pela bibliografia, não devem oferecer riscos à saúde dos consumidores.

Em estudos de teores de nitrato em alface, deve-se observar a metodologia de coleta (hora e parte da planta amostrada) e a análise dos tecidos, pois estas podem afetar decisivamente os resultados.

Ainda existe grande divergência no que tange ao assunto nitrato e à saúde humana, dessa forma, mais estudos clínicos do papel do nitrato no organismo humano devem ser realizados, com o propósito de esclarecer se sua ingestão é benéfica ou prejudicial à saúde.

\section{AGRADECIMENTOS}

Ao Conselho Nacional de Desenvolvimento Científico e Tecnológico (CNPq), à Coordenação de Aperfeiçoamento de Pessoal de Nível Superior (CAPES) e à Fundação de Amparo à Pesquisa do Estado do Rio Grande do Sul (FAPERGS), pelas bolsas de iniciação científica, mestrado e doutorado concedidas, bem como ao CNPq pela Bolsa de Produtividade em Pesquisa.

\section{REFERÊNCIAS}

ADDISCOTT, T.; BENJAMIN, N. Are you taking your nitrate? Food Science and Technology Today, London, v.14, n.1, p.59-61, 2000.

ALVARENGA, M.A.R. et al. Crescimento, teor e acúmulo de macronutrientes em alface americana sob doses de $\mathrm{N}$ aplicados no solo e de níveis de cálcio aplicados via foliar. Horticultura Brasileira, Brasília, v.18, Supl, p.803-804, 2000.

ANDRIOLO, J.L. Fisiologia das culturas protegidas. Santa Maria: UFSM, 1999. 142p.

ARCHER, D.L. Evidence that ingested nitrate and nitrite are beneficial to health. Journal of Food Protection, Des Moines, v.65, n.5, p.872-875, 2002.

Ciência Rural, v.38, n.8, nov, 2008. 
AVERY, A.A. Infantile methaemoglobinaemia: Reexamining the role of drinking water nitrates. Environmental Health Perspectives, North Carolina, n.107, p.583-586, 1999.

BARRET, J.H. et al. Nitrate in drinking water and the incidence of gastric, esophageal, and brain cancer in Yorkshire, England. Cancer Causes \& Control, Oxford, v.9, n.2, p.153-159, 1998

BENINNI, E.R.Y. et al. Teor de nitrato em alface cultivada em sistemas hidropônico e convencional. Horticultura Brasileira, Brasília, v.20, n.2, p.183-186, 2002.

BOON, J.V.D. et al. Growth and nitrate concentration of lettuce as affected by nitrogen and chloride concentration, $\mathrm{NH}_{4}^{+}: \mathrm{NO}_{3}$ ratio and temperature of the recirculating nutrient solution. Journal of Horticultural Science, Kent, v.65, n.3, p.309321, 1990.

CARDENAS - NAVARRO, R. et al. Nitrate accumulation in plants: a role for water. Journal of Experimental Botany, Lancaster, v.50, n.334, p.613-624, 1999.

CASTELLANE, P.D. Nutrição mineral e qualidade de olerícolas folhosas. In: SÁ, M.E.; BUZZETI, S., (Coords.), Importância da adubação na qualidade dos produtos agrícolas. São Paulo: Ícone, 1994. 437p.

CHUN-YUH, Y. et al. Calcium and magnesium in drinking water and the risk of death from breast cancer. Journal of Toxicology and Environmental Health, Part A, London, v.60, n.4, p.231-241 2000.

COMETTI, N.N. et al. Compostos nitrogenados e açúcares solúveis em tecidos de alface orgânica, hidropônica e convencional. Horticultura Brasileira, Brasília, v.22, n.4, p.748-753, 2004.

CORREA, P.; CHEN, V.W. Gastric cancer. Cancer Surveys, New York, n.20, p.55-76, 1994.

FAQUIN, V. et al. Crescimento e concentração de nitrato em alface sob influência da relação $\mathrm{NO}_{3}^{-}: \mathrm{NH}_{4}^{+}$e cloro na solução nutritiva e do horário de colheita. In: REUNIÃO BRASILEIRA DE FERTILIDADE DO SOLO E NUTRIÇÃO DE PLANTAS, 21., 1994, Petrolina, PE. Anais... Petrolina: Sociedade Brasileira de Ciência do Solo, 1994. p.152-153.

FAQUIN, V.; FURTINI NETO, A.E. Acúmulo de nitrato $\left(\mathrm{NO}_{3}{ }^{-}\right)$ em alface: mito? NOTESALQ, Piracicaba, n.5, p.4-5, 1996.

FAQUIN, V.; ANDRADE, A.T. Nutrição mineral e diagnose do estado nutricional de hortaliças. Lavras: UFLA/FAEPE, 2004. 88p.

FERNANDES, A.A. et al. Produtividade, acúmulo de nitrato e estado nutricional de cultivares de alface, em hidroponia, em função de fontes de nutrientes. Horticultura Brasileira, Brasília, v.20, n.2, p.195-200, 2002.

FERREIRA, V.P. et al. Resposta de alface a diferentes épocas de aplicação de N. Horticultura Brasileira, Brasília, v.18, Suplemento, p.791-793, 2000.

FILGUEIRA, F.A.R. Novo manual de olericultura: agrotecnologia moderna na produção e comercialização de hortaliças. Viçosa: UFV, 2000. 402p.
FILGUEIRAS, R.C. et al. Produção de alface hidropônico em diferentes condutividades elétricas. Semina: Ciências Agrárias, Londrina, v.23, n.2, p.157-164, 2002.

FURLANI, P.R. Instruções para o cultivo de hortaliças de folhas pela técnica de hidroponia - NFT. Campinas: IAC, 1998. 30p. (IAC. Boletim Técnico, 168).

HUFTON, C.A. et al. Effects of $\mathrm{NO}\left(+\mathrm{NO}_{2}^{-}\right)$pollution on growth, nitrate reductase activities and associated protein contents in glasshouse lettuce grown hydroponically in winter with $\mathrm{CO}_{2}$ enrichment. New Phytologist, Sheffield, v.133, p.495-501, 1996 .

JOOSSENS, J. et al. Dietary salt, nitrate an stomach cancer mortality in 24 countries. International Journal of Epidemiology, Oxford, n.25, p.494-504, 1996.

KEY, T.J.A. et al. Dietary habits and mortality in 11000 vegetarians and health conscious people: results of a 17 year follow up. British Medical Journal, London, n.313, p.775778,1996

KROHN, N.G. et al. Teores de nitrato em folhas de alface em função do horário de coleta e do tipo de folha amostrada. Horticultura Brasileira, Brasília, v.21, n.2, p.216-219, 2003.

LEIFERT, C. et al. Human health effects of nitrate. In: IFA AGRICULTURAL CONFERENCE ON MANAGING PLANT NUTRITION, 1999, Barcelona. Proceedings... Barcelona: IFA, 1999. p.1-12.

MANTOVANI, J.R. et al. Comparação de procedimentos de quantificação de nitrato em tecido vegetal. Pesquisa Agropecuária Brasileira, Brasília, v.40, n.1, p.53-59, 2005.

MAYNARD, D.N. et al. Nitrate accumulation in vegetables. Advances in Agronomy, New York, v.28, p.71-118, 1976.

McCALL, D.; WILLUMSEN F. Effects of nitrate, ammonium and chloride application on the yield and nitrate content of soil-grown lettuce. Journal of Horticultural Science \& Biotechnology, Kent, v.73, n.5, p.698-703, 1998.

McKNIGHT, G.M. et al. Dietary nitrate in man: friend or foe? British Journal of Nutrition, London, n.81, p.349-358, 1999.

MIRVISH, S.S. Inhibition by vitamins $\mathrm{C}$ and $\mathrm{E}$ of in vivo nitrosation and vitamin $\mathrm{C}$ occurrence in the stomach. European Journal of Cancer Prevention. Hasselt, n.5 suppl.1, p.131-136, 1996.

MONDIN, M. Efeito de sistema de cultivo na produtividade e acúmulo de nitrato em cultivares de alface. 1996. $88 f$. Tese (Doutorado em Agronomia) - Faculdade de Ciências Agrárias e Veterinárias, Universidade Estadual Paulista "Júlio de Mesquita Filho". Jaboticabal.

OHSE, S. Rendimento, composição centesimal e teores de nitrato e vitamina c em alface sob hidroponia. 1999. 103f. Tese (Doutorado em Produção Vegetal) - Escola Superior de Agricultura "Luiz de Queiroz", Universidade de São Paulo. Piracicaba.

OHSE, S. Qualidade nutricional e acúmulo de nitrato em alface. In: SANTOS, O.S. dos. (Org.). Hidroponia da alface. Santa 
Maria: Universidade Federal de Santa Maria, 2000. Cap.2, p.10-24.

PEREIRA, N.N.C. et al. Adubação nitrogenada na cultura da alface fontes de $\mathrm{N}$ e inibidor de nitrificação. Pesquisa Agropecuária Brasileira, Brasília, v.24, n.6, p.647-654, 1989.

PIGNATELLI, B. et al. Mutagens, N-nitroso compounds and their precursors in gastric juice from patients with and without precancerous lesions of the stomach. European Journal of Cancer, Edimburg, v.29A, n.14, p.2031-2039, 1993.

SALSAC, L. et al. Nitrate and ammonium nutrition in plants. Plant Physiology and Biochemistry, Paris, v.25, p.805812, 1987.

SOBALA, G.M. et al. Ascorbic acid in the human stomach. Gastroenterology, Philadelphia, n.97, p.357-363, 1989.

TAIZ, L.; ZIEGER, E. Fisiologia vegetal. (Trad.). SANTAREM E.R. et al. 3.ed. Porto Alegre: Artmed, 2004. 719p.
WARD, M. et al. Nitrate in public water supplies and risk of bladder cancer. Epidemiology, Baltimore n.14, p.183-190, 2003.

WORLD HEALTH ORGANIZATION (WHO). Toxicological evaluation of certain food additives with a review of general principles and of specifications. Seventeenth report of the joint FAO/WHO Expert Committee on Food Additives. FAO Nutrition Report Series, Geneva n.539, p.42, 1973.

WORLD HEALTH ORGANIZATION (WHO). Evaluation of certain food additives. Twentieth meeting of the joint FAO/ WHO Expert Committee on Food Additives. WHO Food Additives Series, Geneva, n.599, p.32, 1976.

WRIGHT, M.J.; DAVISON, K.L. Nitrate accumulation in crops and nitrate poisoning in animals. Advances in Agronomy, New York, v.16, p.197-274, 1964.

ZITO, R.K. et al. Fontes de nutrientes, relações nitrato:amônio e molibdênio, em alface (Lactuca sativa) produzida em meio hidropônico. Revista Ceres, Viçosa, v.41, n.236, p.419-430, 1994. 